\title{
HISTOLOGICAL EVALUATION OF IMMUNE ORGANS IN CHICKEN EMBRYOS INOCULATED WITH MAREK'S DISEASE VIRUS AND LYMPHOKINES
}

\author{
Mireya OrTiz , V. M. Petrone, G. TÉLleZ and T. FehÉRVÁRI \\ Departamento de Producción Animal: Aves, Facultad de Medicina, Veterinaria y \\ Zootecnia, Universidad Nacional Autónoma de México, 04510 Ciudad Universitaria, \\ Coyoacán, México D. F., México
}

(Received September 21, 2000; accepted February 8, 2001)

\begin{abstract}
The aim of the present study was to evaluate the presence of lymphocytes and granulocytes in different stages of embryonic development and on the first posthatching day. The lymphocytes present in the bursa of Fabricius and thymus were evaluated by histological analysis of the yolk sac, bursa of Fabricius, thymus, liver and bone marrow of 100 chicken embryos divided into groups and treated with: (I) Marek's disease vaccine as viral antigen, (II) Marek's disease vaccine plus lymphokines, (III) lymphokines, and (IV) vaccine diluent. Group V was not treated. Samples were taken on days 14, 17 and 20 of incubation and on the first posthatching day. An increase in the number of epithelial matrix as precursors of lymphoid follicles was observed in the bursa of Fabricius of embryos inoculated with lymphokines compared to embryos in all the other groups $(\mathrm{p}<$ 0.05 ). In addition, a higher amount of granulocytes was found in the yolk sac and liver of embryos inoculated with lymphokines than in the embryos of all other groups $(\mathrm{p}<0.05)$. In the bone marrow, no significant difference was observed among the treated groups concerning the amount of granulocytes. The results suggest that administration of antigens or protein molecules at an early stage of embryonic development increases the presence of granulocytes in the liver and granulopoiesis in the yolk sac, and also increases the number of epithelial matrixs in the bursa of Fabricius.
\end{abstract}

Key words: Bone marrow, bursa of Fabricius, histopathology, granulocytes, liver, lymphocytes, lymphokines, Marek's disease vaccine, thymus, yolk sac

The immune system of the chicken embryo is immature during development; however, an unspecific immune system allows embryonic survival. Among the components of this mechanism are dendritic cells, macrophages and granulocytes (Seto, 1981) which can phagocytose bacteria and other particles; embryonic interferon which acts as antiviral agent, and complement which acts through its alternative pathway to cause cytolysis. The yolk sac, aorta, spleen, intestine and liver are the sites of phagocytic activity (Seto, 1981).

"Corresponding author; E-mail: monicolim@yahoo.com; Fax: +52 56166923 
The main organs of the chicken embryo are the yolk sac, thymus and bursa of Fabricius (BF) which produce lymphocyte precursor cells (Seto, 1981). The bird thymus is a paired lymphoid organ with 6-8 pairs of lobules consisting of cortex and medulla. The cortex has lymphocytes and is covered by a connective tissue capsule. The medulla is composed of epithelial and dendritic cells, and few lymphocytes. The BF is a sack with 11 to 17 folds on the inside surface, which are oriented towards the light. These folds have lymphoid follicles. Each follicle has a cortex and a medulla. The BF medulla contains epithelial cells that are replaced by dendritic cells and lymphocytes (Tizard, 1992). The cortex develops after birth and contains lymphocytes, dendritic cells and macrophages (Tizard, 1992). In the spleen and BF, granulopoiesis starts at 7 days of incubation and erythropoiesis at 11 and 15 days of incubation (Glávits et al., 1981). The thymus contains lymphocytes at 10 days of incubation. During this time, the thymus is active in lympho- and granulopoiesis (Seto, 1981).

Evidence has also been found that immunological maturation starts before birth. According to Moore and Owens (1967), germinal haematopoietic cells proceed from the yolk sac, migrate to the bone marrow, and mature. The yolk sac surface is larger from days 6 to 12 of incubation, the stage at which precursor haematopoietic cell proliferation starts. Polymorphonuclear leukocytes are granulocytes and form part of nonspecific immunity in birds and reptiles (Maxwell, 1984). Granulocytes have been found in haematopoietic organs since day 12 of incubation (Zulkifli and Siegel, 1994). Differentiation of B lymphocytes starts between days 7 and 14 of incubation and precursor cells capable of producing immunoglobulin (Ig) M, IgY and IgA can be detected on days 10, 14 and 16 of incubation respectively, while in the thymus $\mathrm{T}$ lymphocytes differentiate, and CD3 lymphocytes can be detected at 9 days of incubation (Sharma et al., 1997).

Activated T lymphocytes release soluble substances called lymphokines (Gómez, 1995). These substances act on many different cell types and induce functional changes: e.g. interleukin-2 (IL-2) participates in the growth and antibody synthesis of $\mathrm{B}$ lymphocytes, while the granulocyte and monocyte colony stimulating factor (GM-CSF) participates in the differentiation of granulocytes among other cells (Abbas et al., 1995). Recent findings show that the prophylactic administration of lymphokines produced from chickens immunized against Salmonella enteritidis phagotype 13 (SE-ILK) confers significant protection against Salmonella spp. infection in chicken embryos at 18 days of incubation and when administered to day-old chicks and turkeys (Kogut et al., 1998). In ovo it is possible that prophylactic protection conferred by lymphokines does not only reduce infectivity of one specific organism, but also of others antigenically different (Kogut et al., 1998). The morphological and cellular effect exerted on lymphoid organs by administration of SE-ILK at early stages of embryonic development has not been determined. The reaction of the immature immune system to an antigenic challenge alone or in conjunction with SE-ILK is not documented. 
Although lymphokines are known to stimulate the immune system by proliferation, differentiation and cell activation (Abbas et al., 1995) among other mechanisms, the effect of lymphokines simultaneously applied with a viral antigen to chicken embryos has not been studied. The aim of the present work was to evaluate cell presence and migration, which contribute to the immune response at various stages of embryonic development and in day-old chicks. Histological analysis of embryonic tissues treated with SE-ILK and viral antigens was used to determinate cell types and the number of cells present in the tissues examined.

\section{Materials and method}

\section{Experimental embryos}

One hundred specific pathogen free embryos of 10 days were used (Alpes ${ }^{\circledR}$, Tehuacán, Puebla, México).

\section{Bivalent vaccine}

The bivalent vaccine (BV) consisted of strains of HVT and SB-1, the commercial vaccine used to evade Marek's disease (MD), with titres of $10^{3}$ plaque forming units (PFU)/dose respectively, of $200 \mu \mathrm{l}$ each (INTERVET $^{\mathbb{R}}$ Toluca, Edo. de México, Mexico).

\section{Vaccine diluent}

The vaccine diluent was the same as used to dilute the bivalent vaccine by INTERVET ${ }^{\circledR}$.

\section{Lymphokines}

These were raised in birds immunized against Salmonella enteritidis phagotype 13 (SE-ILK), prepared and used without further treatment as described by Kogut and Slajchert (1992) and Téllez et al. (1993).

\section{Treatment design}

Embryos were randomly divided into 5 groups of 20 embryos each and inoculated via the yolk sac on day 10 of incubation, as follows:

Group I: Embryos inoculated with a complete dose of $200 \mu \mathrm{l}$ of BV-MD.

Group II: Embryos inoculated with a complete dose of $200 \mu 1$ of BV-MD plus $100 \mu \mathrm{l}$ of SE-ILK.

Group III: Embryos inoculated with $100 \mu \mathrm{l}$ of SE-ILK.

Group IV: Embryos inoculated with $200 \mu$ of vaccine diluent.

Group V: Embryos not inoculated. 


\section{Sampling schedule}

Four samples were taken on days 14, 17 and 20 of embryonic development and 1 day after birth from the yolk sac, liver, $\mathrm{BF}$ and thymus.

\section{Samples}

Embryos were euthanised as described by Andrews et al. (1993). Subsequently they were fixed with pins to a paraffin plate, and the necropsy was performed with a magnifying glass to remove the organs needed.

\section{Histology}

The right thymus chain was taken, a transversal section of $2 \mathrm{~mm}$ width of the middle part of the left liver lobule, and an area of $1 \mathrm{~cm}^{2}$ of the distal pole of the yolk sac, $5 \mathrm{~mm}$ of the femoral bone marrow and the complete BF. The samples were fixed in $10 \%$ formalin, buffered to $\mathrm{pH} 7.4$. Once fixed, the organs were processed by the routine technique for paraffin sections and stained with haematoxylin and eosin (Allen, 1995).

\section{Histological evaluation}

Yolk sac. Ten perivascular areas were selected and transversally sectioned. Cells were counted to obtain the proportion of granulocytes in relation to the rest of the haematopoietic cells (Petrone et al., 1998).

Liver. A transversal section of $2 \mathrm{~mm}$ width of the middle part of the left liver lobule was used to count perivascular areas to obtain the proportion of granulocytes (Petrone et al., 1998).

Bursa of Fabricius. A transversal section of the middle part of each BF was taken to count the number of cumuli of epithelial cells as the matrix for formation of lymphoid tissue in early embryonic life (14 days) and the number of lymphoid follicles later (17, 20 and first posthatching day), per section of each BF.

Thymus. A longitudinal section of the right thymus chain was made to obtain the proportion of the cortex and medullar width. The proportion was expressed as the percentage of the thymic medulla considering the sum in $\mathrm{mm}$ of the cortex and medullar width as $100 \%$.

Bone marrow. Ten microscopic fields were selected to count and obtain the proportion of granulocytes with respect to the rest of haematopoietic cells. This sample was only taken in day-old chicks instead of the yolk sac, which was in the process of involution at that point of development. 

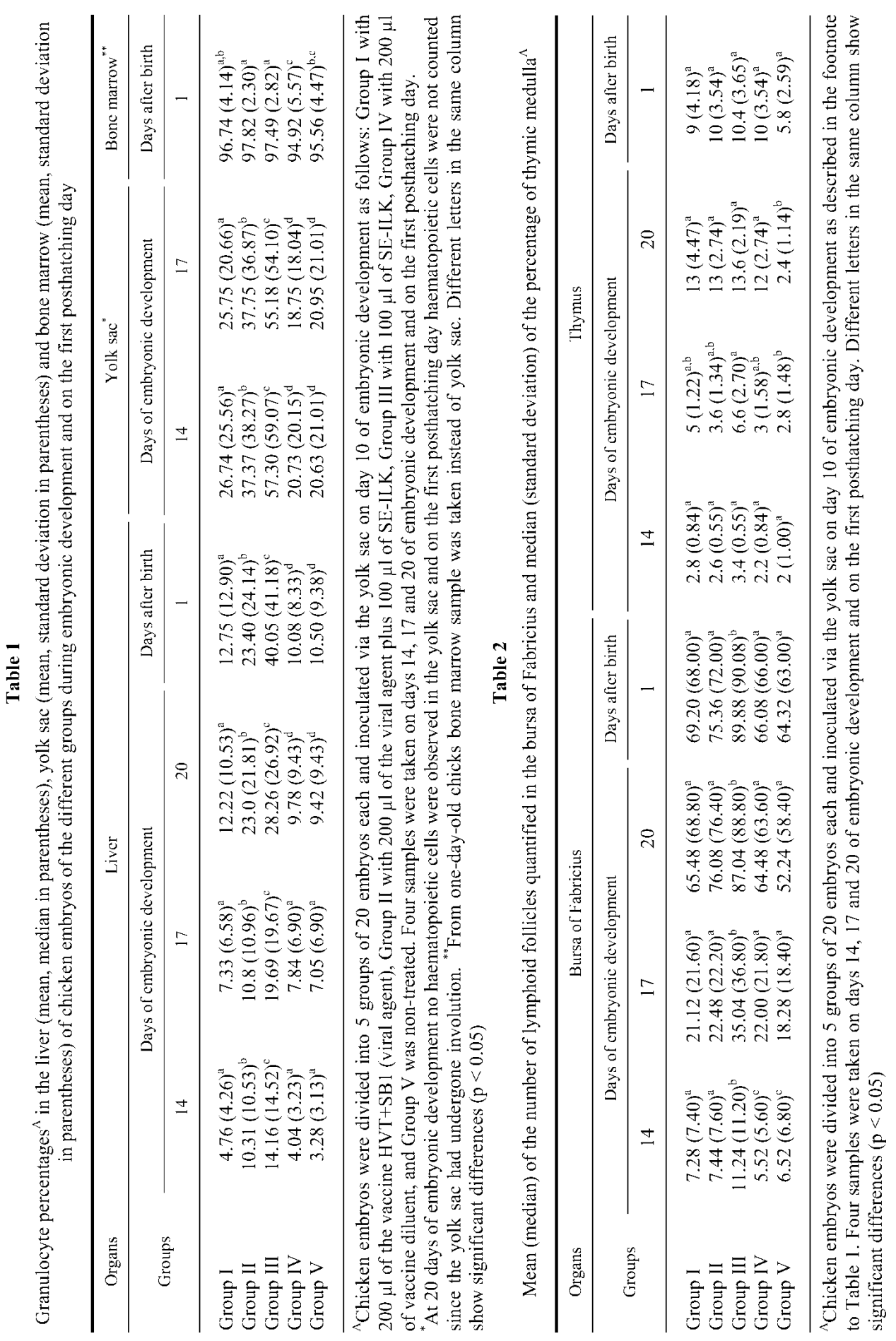
Titration of the bivalent vaccine

A sample of the BV-MD equivalent to a complete dose of $200 \mu \mathrm{l}$ was taken aseptically and processed for titration of avian herpesvirus HVT and SB-1, according to the method by Palya (1991).

\section{Bacteriological control}

Liver, thymus, yolk sac, bone marrow and BF samples were taken and processed for bacterial isolation with routine bacteriological methods (Mallison and Snoeyenbos, 1993) to prove the absence of aerobic bacteria in embryos during the experiment.

\section{Statistical analysis}

Data were transformed by the arc sine of the square root (Wayne, 1987) for statistical analysis of the percentage of granulocytes in bone marrow, in the proportion of thymic cortex and medulla, and in the perivascular areas of the yolk sac. Analysis of variance was applied to transformed data and the difference between means was evaluated by Tukey's test (Marriott, 1974; Méndez, 1981).

The Kruskall-Wallis test was applied for comparison of medians of the spaces of the portal region or areas peripheral to the central lobular veins of the liver with granulocytes, and the amount of lymphoid follicles in the BF. Comparison of all groups was made and differences between groups were analyzed by the Mann-Whitney U test (Marriott, 1974; Méndez, 1981). The level of significance was $\mathrm{p}<0.05$.

\section{Results}

\section{Bivalent vaccine titration}

The presence of Marek's disease viruses (HVT + SB-1) was confirmed by titration in chicken embryo fibroblast culture, at a concentration of $10^{3} \mathrm{PFU} /$ $200 \mu \mathrm{l}$ in a sample taken to inoculate embryos. The samples of lymphokines and vaccine diluent were free of Marek's disease virus as confirmed by isolation.

\section{Granulocyte quantification}

The four samples taken for morphological evaluation (on days 14, 17 and 20 of embryonic development and from day-old chicks) showed a significant increase $(\mathrm{p}<0.05)$ in the mean percentage of granulocytes in the liver and yolk sac of embryos inoculated with SE-ILK (Group III), followed by the group inoculated with the bivalent vaccine plus SE-ILK (Group II) (Table 1 and Fig. 1). In the group of embryos which received viral antigen (Group I), a significant increase was observed in the number of granulocytes present in the yolk sac on 
days 14 and 17 of embryonic development $(p<0.05)$. No cellular differences were found in the liver in any evaluation $(p>0.05)$. Embryos of groups inoculated with vaccine diluent (Group IV) and the negative control (Group V) always exhibited a lower number of granulocytes in the yolk sac and liver $(p>0.05)$ (Table 1 and Fig. 1).

\section{Quantification of lymphoid follicles}

Of the four samples taken for morphologic evaluation (on days 14, 17 and 20 and from one-day chicks), the median of lymphoid follicles was greater in embryos inoculated with SE-ILK (Group III) $(\mathrm{p}<0.05)$ (Table 2, Fig. 2). Treatment with viral agent (Group I) and with viral agent plus SE-ILK (Group II) increased $(p<0.5)$ the amount of follicles. This increase was observed from day 10 of inoculation until day 14 of embryonic development. After day 17 of embryonic development no significant difference was found between the groups mentioned above and embryos inoculated with vaccine diluent (Group IV) and negative controls (Group V). The amount of follicles increased from day $11 \mathrm{of}$ embryonic development in all groups.

\section{Quantification of thymic medulla percentage}

The thymus showed the least expressed reaction to the different treatments (Table 2). In thymus samples taken on days 14, 17 and 20 of embryonic development and from one-day chicks no significant difference was seen among the inoculated groups (Group I to Group V) in the proportion of medulla and cortex $(p>0.05)$. However, in the group inoculated with SE-ILK (Group III), the proportion of medulla was numerically the highest of all groups.

\section{Bacteriological control}

Organs subjected to bacteriological analysis gave negative result, showing that no bacterial contamination occurred during the study.

\section{Discussion}

The results show that all treatments had an effect on one or more of the parameters measured in the experiment. The effects were dramatic in the SE-ILK group (Group III) and less evident in the vaccine diluent group (Group IV) (Tables 1 and 2, Figs 1 and 2).

The present investigation showed that the treatment significantly increased the number of granulocytes compared with untreated embryos. This effect correlated with age, i.e., granulocyte number increased with embryonic development although treatments were applied at early stages. The number of granulocytes 

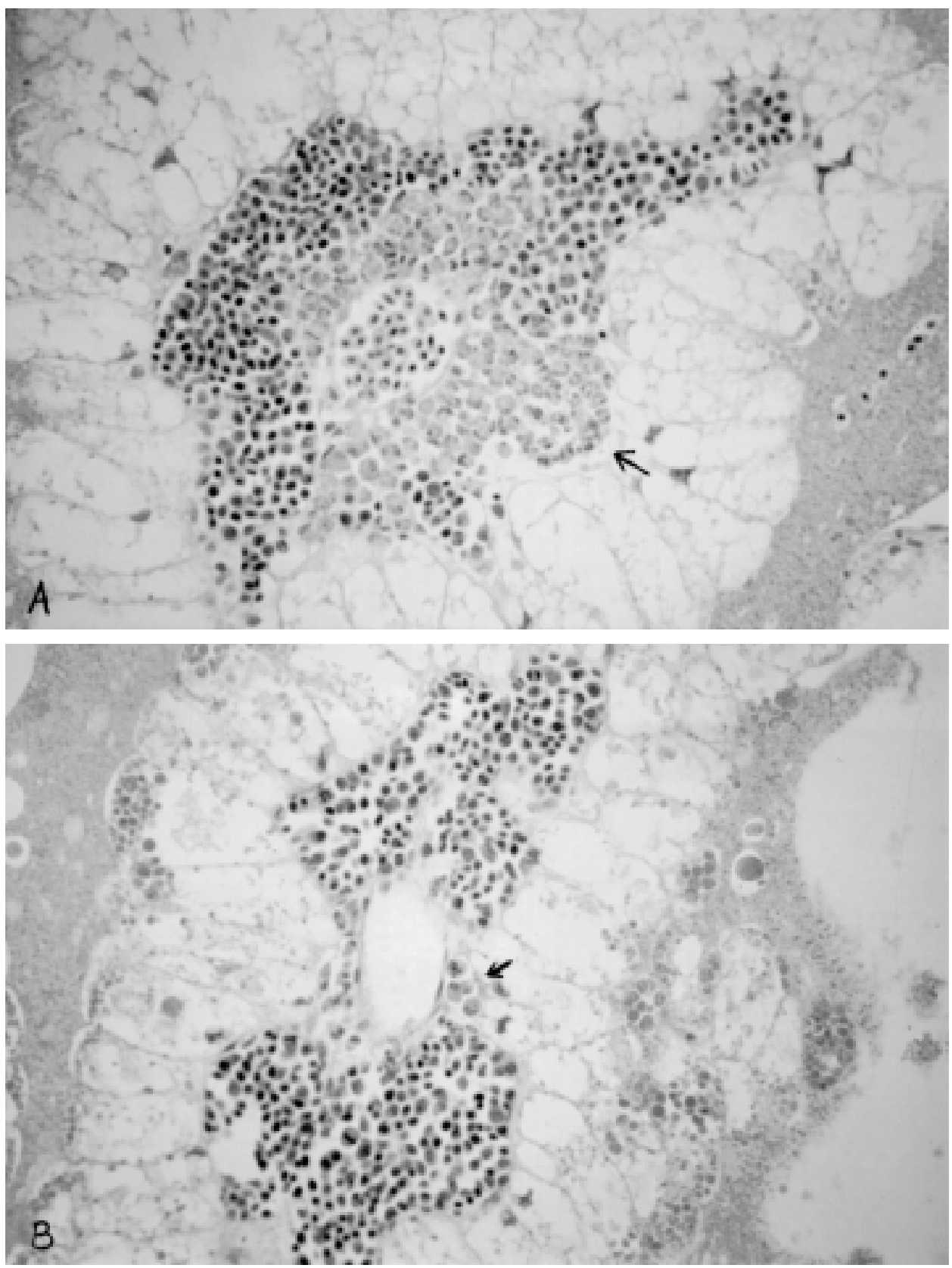

Fig. 1. (A) Increased number of granulocytes $(\rightarrow$ ) in the yolk sac of 13-day-old embryos inoculated with SE-ILK. (B) Development of granulocytes $(\rightarrow)$ in the yolk sac of 13-day-old non-inoculated embryos. Haematoxylin and eosin, $\times 400$ 

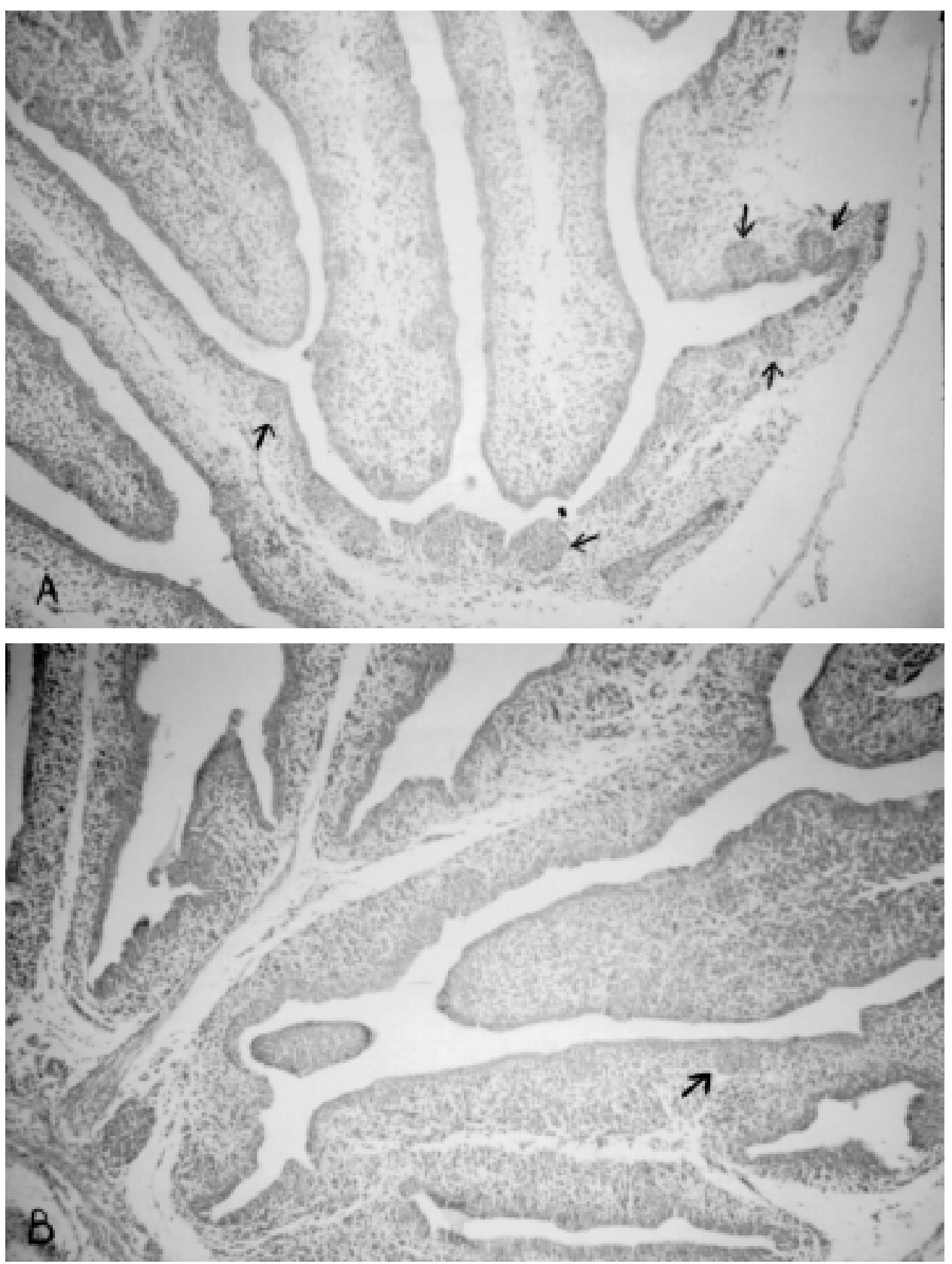

Fig. 2. (A) Increase in the amount of lymphoid follicles $(\rightarrow)$ in the bursa of Fabricius of 13-day-old chicken embryos inoculated with SE-ILK. (B) Development of lymphoid follicles $(\rightarrow$ ) in the bursa of Fabricius of 13-day-old non-inoculated chicken embryos. Haematoxylin and eosin, $\times 400$ 
increased in the yolk sac (Fig. 1) and granulocytes appeared in the liver (Table 1). This result confirms that granulopoiesis may be stimulated at early stages by the presence of lymphokines and by viral antigen (BV-MD) or both. However, the greatest stimulating effect of SE-ILK occurred in the liver and yolk sac. In the bone marrow, granulocytes occurred in higher numbers in the group that received viral antigen plus SE-ILK (Group II) than in the group that received SEILK only (Group III, Table 1). This agrees with the report by Kogut et al. (1998), who found that administration of SE-ILK confers significant protection against infection with Salmonella spp. if applied to 18-day-old embryos or to one-dayold chicks or turkeys.

Studies by McGruder et al. (1993) and Kogut et al. (1996) show that lymphokines administered during the last days of incubation increase granulocyte proliferation, mainly of the heterophils. The present work reveals that granulopoiesis can be stimulated if SE-ILK is administrated as early as day 10 of embryonic development. The positive effect of SE-ILK administration was detected by day 14 of embryonic development in the yolk sac and liver; in the liver an increase in the number of granulocytes was observed throughout the study (Table 1). The same positive effect was maintained in the presence of viral antigen, since the number of granulocytes observed in embryos inoculated with viral antigen plus SE-ILK (Group II) was significantly higher $(p<0.05)$ than in the negative control (Group V) and in the group inoculated with viral antigen (Group I, Table 1).

In addition to its stimulating effect on granulopoiesis, administration of SE-ILK also increased the amount of lymphoid follicles significantly in the BF, which represents a greater amount of lymphocytes (Table 2, Fig. 2). Such an increase was not detected in the other groups, not even in the group that received antiviral antigen plus SE-ILK (Group II, Table 2).

No significant changes were observed in the thymus (Table 2), since SEILK only produced detectable activation by the histological technique applied to the bursal B-lymphocytes (Table 2, Fig. 2).

Results of the present study suggest that the administration of antigens or protein molecules at an early stage, i.e. at 10 days of embryonic development, is capable of producing increased granulopoiesis in the yolk sac, as well as a rise in the number of lymphoid follicles in the bursa of Fabricius and the appearance of granulocytes in liver (Tables 1 and 2, Figs 1 and 2).

\section{Acknowledgements}

Thanks are expressed to Dr. Michel Kogut for providing the lymphokines and to Virginia Lowry for critical review of the paper, both of them from the Agricultural Research Service, Food Animal Protection Research Laboratory, U.S. Department of Agriculture, Texas, USA. 


\section{References}

Abbas, A. K., Lichtman, A. H. and Pober, J. S. (1995): Cellular and Molecular Immunology (in Spanish). 2nd edition. Interamericana McGraw-Hill, Madrid (Spain).

Allen, T. C. (1995): Hematoxylin and Eosin. In: Prophet, E. B., Mills, B., Arrington, J. B. and Sobin, L. H. (eds) Histotechnical Methods (in Spanish). Institute of Pathology for Armed Forces of the United States of America, Washington, D.C. pp. 55-60.

Andrews, E. J., Bennett, B. T., Clark, J. D., Houpt, K. A., Pascoe, P. J., Robinson, G. W. and Boyce, J. R. (1993): Report of the AVNA Panel on Euthanasia. JAVNA 202, 229-249.

Daniels, W. (1987): Biostatistics, Basis for the Analysis of Health Science (in Spanish). 3rd edition. Limusa, Mexico City.

Glávits, R., Rátz, F. and Fehérvári, T. (1981): The role of the bursa of Fabricius in the ontogeny of the myeloid system. Acta Vet. Acad. Sci. Hung. 29, 327-333.

Gómez, V. G. (1995): Identification of lymphokines supernatant in chicken splenocytes stimulated with concanavalin A (in Spanish). Thesis, Faculty of Veterinary Medicine and Zootechnics, UNAM, Mexico City.

Kogut, M. H. and Slajchert, T. B. (1992): T lymphocytes confer protection in chickens against Eimeria tenella by production of lymphokines. Immun. Infect. Dis. 2, 69-80.

Kogut, M. H., Téllez, I. G., McGruder, E. D., Wong, R. A., Isibasi, A., Ortiz, V. N., Hargis, B. M. and DeLoach, J. R. (1996): Evaluation of Salmonella enteritidis immune lymphokines on host resistance to Salmonella enterica ser. gallinarum infection in broiler chicks. Avian Pathol. 25, 237-249.

Kogut, H. M., Wells, L. L. and Lowry, K. V. (1998): Ontogeny of bactericidal and phagocytic activity of heterophil cells of chicken and turkey and their lymphocytic stimulation (in Spanish). Proceedings of the VIIth Poultry Medicine Symposium, 11-12 March 1998, Mexico City, Mexico. National Association of Poultry Science Specialists. pp. 44-47.

Mallison, E. T. and Snoeyenbos, G. H. (1993): Salmonellosis. In: Hitchner, B. S. (ed.) A Laboratory Manual for the Isolation and Identification of Avian Pathogens. 3rd edition. The American Association of Avian Pathologists, USA.

Marriott, F. H. C. (1974): The Interpretation of Multiple Observations. Academic Press, London, U. K.

Maxwell, M. H. (1984): Histochemical identification of tissue eosinophils in the inflammatory response of the fowl (Gallus domesticus). Res. Vet. Sci. 37, 7-11.

McGruder, E. D., Ray, P. M., Téllez, I. G., Kogut, M. H., Corrier, D. E., Deloach, J. R. and Hargis, B. M. (1993): Salmonella enteritidis immune leukocyte-stimulated soluble factors: effects on increased resistance to Salmonella organ invasion in day-old Leghorn chicks. Poultry Sci. 72, 2264-2271.

Méndez, R. I. (1981): Statistical Linear Models (in Spanish). 2nd edition. CONACYT, Mexico City, Mexico.

Moore, M. A. S. and Owen, J. J. T. (1967): Stem cell migration in developing myeloid systems. Lancet 2, 658-659.

Palya, V. (1991): Manual for the Production of Marek's disease, Gumboro Disease and Inactivated Newcastle Disease Vaccines. Food and Agriculture Organization Animal Production and Health Paper. 1st ed., Rome.

Petrone, V. M., Santiago, R., Escorcia, M., Fehérvári, T., Juárez, M. A. and Téllez, G. (1998): Evaluation of the leukocyte response in chicken embryos inosulated with herpesvirus type 1 (in Spanish). Proceedings of the VIIth National Congress of Veterinary Pathology, 3-6 June 1998, Manzanillo (Colima). Veterinary Pathologist Society AC, Mexico City, Mexico, pp. 12-14.

Seto, F. (1981): Early development of the avian immune system. Poultry Sci. 60, 1981-1995. 
Sharma, J. M. (1997): The structure and function of the avian immune system. Acta Vet. Hung. 45, 229-238.

Téllez, I. G., Kogut, M. H. and Hargis, B. M. (1993): Immunoprophylaxis of Salmonella enteritidis infection by lymphokines in Leghorn chicks. Avian Dis. 37, 1062-1070.

Tizard, I. (1992): Veterinary Immunology (in Spanish). Interamericana McGraw-Hill, Mexico City, Mexico.

Zulkifli, I. and Siegel, P. B. (1994): Heterophil to lymphocyte ratios during perinatal and neonatal stages in chickens. Br. Poult. Sci. 35, 309-313. 